Article

\title{
Evaluation of Traditional Chinese Medicinal Plants for Anti-MRSA Activity with Reference to the Treatment Record of Infectious Diseases
}

\author{
Guo-Ying Zuo ${ }^{1{ }_{*},}$, Xin-Juan Zhang ${ }^{1,2, \dagger}{ }^{,}$, Cui-Xian Yang ${ }^{1,3, \dagger}$, Jun Han ${ }^{3}$, Gen-Chun Wang ${ }^{1}$ and \\ Zhong-Qi Bian ${ }^{4, *}$
}

1 Research Center for Natural Medicines, Kunming General Hospital, PLA, Kunming 650032, China; E-Mails: shmily_zxj2008@163.com (X.-J.Z.); ycx484@126.com (C.-X.Y.);

kmwgc12@126.com (G.-C.W.)

2 Kunming Medical College, Kunming 650032, China

3 Yunnan Traditional Chinese Medical College, Kunming 650500, China; E-Mail: hanzjn@126.com

4 Center for Infectious Diseases, Kunming General Hospital, PLA, Kunming 650032, China

$\dagger$ These authors contributed equally to this work.

* Authors to whom correspondence should be addressed; E-Mails: zuoguoying@263.net (G.-Y.Z.); bzq@ynu.edu.cn (Z.-Q.B.); Tel./Fax: +86-871-477-4941 (G.-Y.Z.).

Received: 8 February 2012; in revised form: 27 February 2012 / Accepted: 29 February 2012 / Published: 9 March 2012

\begin{abstract}
The in vitro antimicrobial activities of 30 Chinese medicinal plants were evaluated with reference to the treatment record of infectious diseases in the Traditional Chinese Medicine (TCM) literature. The plant materials were extracted with $80 \%$ ethanol and the extracts were primarily screened against conventional clinical pathogens like Staphylococcus aureus, Escherichia coli, Pseudomonas aeruginosa and Candida albicans by the agar diffusion method. Their inhibition zone diameters (IZDs, mm, $50 \mathrm{mg} / \mathrm{mL}$ ) ranged from $<8$ to 24 . The 21 extracts which showed IZDs $\geq 10 \mathrm{~mm}$ against MSSA were also active against methicillin-resistant $S$. aureus (MRSA) with lower IZDs of 9.0-18.8 mm. They were further subjected to minimal inhibitory concentration and minimal bactericidal concentration (MIC/MBC, $\mu \mathrm{G} / \mathrm{mL}$ ) assays, which were $8-2,048 / 32->2,048$ by the standard broth microdilution method. The seven extracts from M. yunnanensis, S. sinensis, G. morella, E. daneillii, M. squamulata, S. arborescens and B. hancei were determined as the most active extracts, with MICs of $8-64 \mu \mathrm{g} / \mathrm{mL}$. The results were in good agreement with their traditional applications in skin and other infections.
\end{abstract}


Keywords: Chinese medicinal plants; traditional Chinese medicine (TCM); methicillin-resistant Staphylococcus aureus (MRSA); inhibition zone diameters (IZD); minimal bactericidal concentrations (MIC)

\section{Introduction}

Staphylococcus aureus infections range from common skin infections, such as furunculosis and impetigo, to severe deep-seated infections. S. aureus ranks first or second among bacterial pathogens causing bloodstream infections. It is the leading cause of nosocomial pneumonia and it also causes infections of surgical wounds and prosthetic implants. Clinical isolates of methicillin-resistant Staphylococcus aureus (MRSA) have become the most common cause of infections among pathogenic bacteria around the Globe, and many life-threatening diseases such as endocarditis, pneumonia and toxin shock syndrome are ascribed to them. Contrary to methicillin-susceptible S. aureus (MSSA), MRSA tend to be multi-drug resistant (MDR), that is, resistant not only to $\beta$-lactam antibiotics but also to a wide range of different antibiotic classes, such as fluoroquinolones, tetracyclines, macrolides, lincosamides and aminoglycosides, and even strains of vancomycin intermediate susceptible or full resistant (VISA and VRSA, respectively) have emerged [1]. Therefore, the search for novel anti-MRSA agents is urgently needed. Meanwhile, great emphasis has been placed on the value of plants used in ethnomedicine and traditional medicine for drug discovery has currently been laid greater stress worldwide [2-5].

Traditional Chinese medicines (TCM), like Ayurveda, Unani and Kampo, have flourished as systems of medicine in use for thousands of years [2]. With more than 5,000 years of Chinese history and as a part of Chinese culture, TCM mainly used plant materials in organized traditional medical systems which have been playing a critical role in fighting various diseases and maintaing human health. Thousands of Chinese herbal medicines have been recorded in a great variety of national and local literatures such as Shen Nong's Materia Medica [6], Compendium of Materia Medica [7], Dictionary of Chinese Materia Medica [8], Chinese Materia Medica [9], Compilation of Chinese Herbal Medicine [10] and Selected Yunnan Traditional Chinese Herbs [11] from ancient to modern times. They have been widely used throughout the country or in local areas by the Chinese people of all nationalities.

In TCM clinical practice, the Chinese disease name "Chuang-Yang" (pyogenic infection and ulceration of skin) is a general terminology for the surgical and skin disease visible over the body surface and dermatosis, including "Yong Ju, Ding-Chuang, Jie-Zhong, Liu-Zhu, Liu-Tan, etc." (carbuncle, deep-seated sore, furuncle, multiple abscess, tuberculosis of bone and joint, scrofula, etc.). The diseases are commonly seen in clinic, due to stagnation of $q i$ and blood stasized from the attack of evils and consumption of blood by heat-evil [12].

Many Chinese herbal medicines (or medicinal plants) have been documented as treatment of these diseases, which prompted us to investigate their inhibitory activity against MRSA. We herein report the in vitro anti-MRSA effects of a collection of 30 Chinese medicinal plants with reference to the treatment record of skin and other kinds of infections (Table 1). 
Table 1. Traditional indications and phytohemical constitution of the 30 Chinese plants.

\begin{tabular}{|c|c|c|c|}
\hline No. & Species & Traditional Indication & Phytohemical Constitution \\
\hline 1 & A. nepalensis & bleeding of the nose, enteritis and dysentery & $\begin{array}{l}\text { tannins, triterpenoids, flavonoids, } \\
\text { phenols }\end{array}$ \\
\hline 2 & B. balsamifer & $\begin{array}{l}\text { anti-rheumatism, ringworm and sores, dysentery, } \\
\text { detoxification and snake bite }\end{array}$ & flavonoids, simple terpenoids \\
\hline 3 & B. hancei & $\begin{array}{l}\text { heat clearing and detoxicating, dysentery, } \\
\text { jaundice, boils, swelling, tuberculosis injury } \\
\text { hematemesis, osteomyelitis, periostitis, } \\
\text { rheumatism and pain }\end{array}$ & $\begin{array}{l}\text { hydroxytyrosol derivatives and } \\
\text { glycosides }\end{array}$ \\
\hline 4 & C. austroglauca & $\begin{array}{l}\text { astringing sores, carbuncles, dysentery, } \\
\text { hemostasis and vaginal discharge }\end{array}$ & none \\
\hline 5 & C. japonica & $\begin{array}{l}\text { carbuncles, boils, mumps, erysipelas, } \\
\text { rheumatism, jaundice, dysentery, hematuria and } \\
\text { gonorrhea }\end{array}$ & $\begin{array}{l}\text { flavonoids (delphinidin } \\
\text { glycosides) }\end{array}$ \\
\hline 6 & C. orbiculatus & $\begin{array}{l}\text { dysentery, multiple abscess, Herpes zoster, } \\
\text { detoxification, inflammatory, cellulites and snake } \\
\text { bite }\end{array}$ & sesquiterpene, flavonoids \\
\hline 7 & C. orchioides & diarrhea, ulcer, pus and muscles atrophy & $\begin{array}{l}\text { triterpenoids, lignans, flavonoids, } \\
\text { alkaloids, stereoids }\end{array}$ \\
\hline 8 & C. prainii & antipyresis, diuretic and chyluria & $\begin{array}{l}\text { alkaloids, polyphenols, } \\
\text { flavonoids }\end{array}$ \\
\hline 9 & E. Burm & $\begin{array}{l}\text { heat clearing and detoxicating, pharyngitis, } \\
\text { dysentery, diarrhea, furuncle ulcer, skin itching, } \\
\text { swelling and pain of hemorrhoids, throat red and } \\
\text { swollen, bleeding gums and traumatic injury. }\end{array}$ & $\begin{array}{l}\text { quinones, triterpenoids, } \\
\text { flavonoids }\end{array}$ \\
\hline 10 & E. daneillii & diarrhea, abdominal pain and vomiting & $\begin{array}{l}\text { alkaloids, flavonoid glycosides, } \\
\text { flavaprin, limonoids }\end{array}$ \\
\hline 11 & E.fortunei & $\begin{array}{l}\text { chronic diarrhea, dysentery, dispersing blood } \\
\text { stasis and traumatic bleeding }\end{array}$ & $\begin{array}{l}\text { alkaloids, triterpenoids, } \\
\text { flavonoids }\end{array}$ \\
\hline 12 & E. laxiflorus & $\begin{array}{l}\text { pesticide, rheumatism, bone fractures and } \\
\text { hemoptysis }\end{array}$ & $\begin{array}{l}\text { alkaloids, triterpenoids, } \\
\text { flavonoids, sesquiterpenes } \\
\text { (agarofurans) }\end{array}$ \\
\hline 13 & G. morella & $\begin{array}{l}\text { wound rot, carbuncle, tinea, ulcer and sore, } \\
\text { anthelminthic and containing toxic substances }\end{array}$ & $\begin{array}{l}\text { phenols (gambogic acid), } \\
\text { flavonoids (xanthones), } \\
\text { triterpenoids }\end{array}$ \\
\hline 14 & I. simonsii & $\begin{array}{l}\text { scabies, bladder hernias, mixed cropping of edible } \\
\text { spices and containing toxic substances }\end{array}$ & $\begin{array}{l}\text { terpenoids, lignans, flavonoids, } \\
\text { phenols }\end{array}$ \\
\hline 15 & K. angustifolia & $\begin{array}{l}\text { anti-infection, swelling and pain, ulcer and } \\
\text { enteritis and heat stroke }\end{array}$ & lignans, triterpenoids \\
\hline 16 & L. lancifolia & stomach pain, vomiting and swelling & $\begin{array}{l}\text { alkaloids, terpenoids, flavonoids, } \\
\text { essential oils }\end{array}$ \\
\hline 17 & M. hongheensis & $\begin{array}{l}\text { vomiting, diarrhea, dysentery, constipation and } \\
\text { geriatric hacking cough }\end{array}$ & alkaloids \\
\hline 18 & M. salicina & carbuncle, furunculosis and sore pain & alkaloids, lignans \\
\hline
\end{tabular}


Table 1. Cont.

\begin{tabular}{|c|c|c|c|}
\hline No. & Species & Traditional Indication & Phytohemical Constitution \\
\hline 19 & M. squamulata & $\begin{array}{l}\text { scabies, carbuncle boils swollen poison, } \\
\text { hemorrhoids, enterobiasis, beriberi, rheumatoid } \\
\text { and snake bite }\end{array}$ & triterpenoids \\
\hline 20 & M. yunnanensis & $\begin{array}{l}\text { hepatitis, sore, otitis media, stomach and } \\
\text { duodenal ulcer, enlarged spleen and boils } \\
\text { swelling, hematuria leucorrhea and traumatic } \\
\text { bleeding }\end{array}$ & $\begin{array}{l}\text { Polyphenols,tannins, } \\
\text { flavonoids,coumarins, various } \\
\text { terpenoids }\end{array}$ \\
\hline 21 & O. javanica & $\begin{array}{l}\text { lump in the abdomen, boils and swelling of throat } \\
\text { and containing toxic substances }\end{array}$ & $\begin{array}{l}\text { falcarindiol, carotatoxin, } \\
\text { 5-allylpyrogallol }\end{array}$ \\
\hline 22 & P. edulis & (no record) & unsaturated organic acids \\
\hline 23 & P. molle & carbuncle, swollen abscess, fistula and scrofula & tannins, flavonoids, alkaloids \\
\hline 24 & R.japonicus & $\begin{array}{l}\text { subsiding swelling, jaundice, malaria lymph node } \\
\text { tuberculosis and pterygium }\end{array}$ & $\begin{array}{l}\text { lactones (anemonin, } \\
\text { protoanemonin), flavonoids }\end{array}$ \\
\hline 25 & S. arborescens & $\begin{array}{l}\text { HBV (skimmianine), rheumatoid, paralysisa, } \\
\text { beriberi, and containing toxic substances }\end{array}$ & $\begin{array}{l}\text { alkaloids, coumarins, } \\
\text { triterpenoids, phenols }\end{array}$ \\
\hline 26 & S. davidii & $\begin{array}{l}\text { antipyretic, detoxicate, subsiding swelling, } \\
\text { laryngitis, pneumonia, dysentery, cystitis and edema }\end{array}$ & polyphenols \\
\hline 27 & S. parasitica & anti-rheumatism and anticancer & flavonoids \\
\hline 28 & S. sinensis & furuncle and swelling & $\begin{array}{l}\text { benzoquinone, tannins, phenols, } \\
\text { lignans, flavonoids, triterpenoids }\end{array}$ \\
\hline 29 & S. tamariscina & $\begin{array}{l}\text { inflammation, pharyngolaryngitis and } \\
\text { bacteriostasis }\end{array}$ & $\begin{array}{l}\text { flavonoids, phenol glycosides, } \\
\text { trehalose }\end{array}$ \\
\hline 30 & S. viridis & urticaria, herpes zoster, rheumatism and analgesia & lignans, triterpenoids, organic acids \\
\hline
\end{tabular}

\section{Results and Discussion}

\subsection{Results}

Table 1 shows the recorded TCM indications and phytohemical constitution of the 30 Chinese medicinal plants [6-11]. The antibacterial susceptible spectrum of the tested seven MRSA strains is listed in Table 2. The initial screening results of the 30 plant extracts against MSSA and other conventional standard strains of Escherichia coli (ATCC 25922), Pseudomonas aeruginosa (ATCC 27853) and Candida albicans (ATCC Y0109) are expressed as inhibition zone diameters (IZDs) in Table 3, while anti-MRSA activities of the selected 21 extracts which were active with IZDs $\geq 10 \mathrm{~mm}$ against MSSA were shown in Table 4. Their corresponding MICs/MBCs $(\mu \mathrm{g} / \mathrm{mL})$ are shown in Table 5.

Table 2. The antibacterial agent-susceptibility test results of MRSA strains.

\begin{tabular}{llll}
\hline MRSA Strains & Resistant (R) & Intermediate (I) & Susceptible (S) \\
\hline \multirow{2}{*}{ MRSA 008 } & PEN, OXS, AMP, LEV, CTX, PIP/S, ERY, CLI, & NONE & VAN, TEC, LNZ, FOS, \\
MRSA 082 & AZM, RIF & FOS & LNZ, VAN \\
MRSA 092 & AZM, CLI, RIF, CTX V, CIP, LEV, CAZ & NONE & COT, RIF, PIP/S, VAN, \\
\end{tabular}


Table 2. Cont.

\begin{tabular}{|c|c|c|c|}
\hline MRSA Strains & Resistant (R) & Intermediate (I) & Susceptible (S) \\
\hline MRSA 123 & PEN, OXS, FOX, IPM CTS & NONE & LNZ, VAN, MXF, FOS \\
\hline MRSA 144 & $\begin{array}{l}\text { PEN, AMP, OXS, FOX, FUR, CFZ, AZM, RIF, } \\
\text { CLI, CLR }\end{array}$ & NONE & VAN, FOS \\
\hline MRSA 189 & $\begin{array}{l}\text { PEN, OXS, AMP, FOX, FUR, CTX, COT, CIP, } \\
\text { LEV }\end{array}$ & FOS & VAN, LNZ, MXF \\
\hline MRSA 321 & $\begin{array}{l}\text { CLR, CLI, AZM, PEN, OXS, AMP, ERY. CTX, } \\
\text { FUR, LEV }\end{array}$ & CAT & VAN, GAT, PIP/T \\
\hline
\end{tabular}

AMP: ampicillin; AZM: azithromycin; CAT: cefathiamidine; CAZ: ceftazidime; CFZ: cefazolin; CIP: ciprofloxacin; CLI: clindamycin; CLR: clarithromycin; COT: Cotrimoxazole; CTS: cilastatin sodium; CTX: Cefotaxime; ERY: erythromycin; FOS: fosfomycin; FOX: cefoxitin; FUR: cefuroxime; GAT: gatifloxacin; IPM: imipenem; LEV: levofloxacin; LNZ: linezolid; MXF: moxifloxacin; OXS: oxacillin; PEN: Penicillin; PIP: piperacillin; PIP/S: piperacillin/sulbactam; PIP/T: piperacillin/ tazobactam; RIF: rifampin; TEC: teicoplanin; VAN: Vancomycin.

Table 3. Screening results of the antimicrobial activities of the extracts from 30 Chinese medicinal plants (IZD: $\mathrm{mm})^{\mathrm{a}}$.

\begin{tabular}{|c|c|c|c|c|c|c|c|c|}
\hline No. & Species & Part $^{b}$ & Weight $(g)^{c}$ & Ratio (\%) ${ }^{\mathrm{d}}$ & $\mathbf{S A}^{\mathrm{e}}$ & $\mathbf{E C}^{\mathrm{f}}$ & $\mathbf{P A}^{\mathrm{g}}$ & $\mathbf{C A}^{\mathrm{h}}$ \\
\hline 1 & A. nepalensis & TBL & 3.85 & 7.7 & 14 & $<8$ & 12 & 9 \\
\hline 2 & B. balsamifer & WP & 0.61 & 1.2 & 12 & 9 & $<8$ & 5 \\
\hline 3 & B. hancei & WP & 4.96 & 9.9 & 19 & 10 & 13 & 11 \\
\hline 4 & C. austroglauca & TBL & 1.50 & 3.0 & 23 & 14 & 15 & 9 \\
\hline 5 & C. japonica & $\mathrm{V}$ & 1.57 & 3.1 & 9 & 11 & 13 & 8 \\
\hline 6 & C. orbiculatus & $\mathrm{V}$ & 2.06 & 4.1 & 11 & 10 & $<8$ & 10 \\
\hline 7 & C. orchioides & WP & 2.28 & 4.6 & 16 & 13 & 14 & 8 \\
\hline 8 & C.prainii & WP & 1.96 & 3.9 & 10 & 10 & $<8$ & 9 \\
\hline 9 & E. Burm & $\mathrm{L}$ & 3.03 & 6.1 & 10 & 10 & 10 & $<8$ \\
\hline 10 & E. daneillii & TBL & 2.52 & 5.0 & 18 & 12 & 10 & 9 \\
\hline 11 & E.fortunei & $\mathrm{V}$ & 4.42 & 8.8 & 15 & 13 & 12 & 11 \\
\hline 12 & E. laxiflorus & TBL & 2.68 & 5.4 & $<8$ & $<8$ & $<8$ & 10 \\
\hline 13 & G. morella & WP & 7.38 & 14.8 & 17 & 12 & $<8$ & $<8$ \\
\hline 14 & I. simonsii & TBL & 3.48 & 7.0 & 13 & 11 & 11 & 8 \\
\hline 15 & K. angustifolia & TBL & 0.99 & 2.0 & $<8$ & 8 & $<8$ & 8 \\
\hline 16 & L. lancifolia & TBL & 2.28 & 4.6 & $<8$ & $<8$ & $<8$ & $<8$ \\
\hline 17 & M. hongheensis & TBL & 3.09 & 6.2 & 21 & 11 & 13 & $<8$ \\
\hline 18 & M. salicina & TBL & 2.91 & 5.8 & 12 & 9 & 8 & 10 \\
\hline 19 & M. squamulata & TBL & 1.48 & 2.9 & 17 & 12 & 12 & 11 \\
\hline 20 & M. yunnanensis & TBL & 2.91 & 5.8 & 24 & 12 & $<8$ & 9 \\
\hline 21 & O. javanica & WP & 2.65 & 5.3 & $<8$ & 8 & $<8$ & $<8$ \\
\hline 22 & P. edulis & TBL & 4.49 & 9.0 & $<8$ & $<8$ & $<8$ & $<8$ \\
\hline 23 & P. molle & WP & 2.99 & 6.0 & 11 & 10 & 16 & 12 \\
\hline 24 & R. japonicus & WP & 5.24 & 10.5 & $<8$ & 9 & 8 & $<8$ \\
\hline 25 & S. arborescens & TBL & 1.86 & 3.7 & 24 & 13 & 14 & 11 \\
\hline 26 & S. davidii & TBL & 2.54 & 5.1 & $<8$ & 8 & 9 & 10 \\
\hline 27 & S. parasitica & TBL & 1.04 & 2.1 & $<8$ & $<8$ & $<8$ & $<8$ \\
\hline
\end{tabular}


Table 3. Cont.

\begin{tabular}{lllllllll}
\hline No. & Species & Part $^{\mathrm{b}}$ & ${\text { Weight }(\mathrm{g})^{\mathrm{c}}}^{\mathrm{c}}$ & Ratio $(\%)^{\mathrm{d}}$ & SA $^{\mathrm{e}}$ & EC $^{\mathrm{f}}$ & PA $^{\mathrm{g}}$ & $\mathbf{C A}^{\mathrm{h}}$ \\
\hline 28 & S. sinensis & TBL & 6.49 & 13.0 & 18 & 8 & 13 & 21 \\
29 & S. tamariscina & WP & 4.44 & 8.9 & 12 & 9 & 11 & 9 \\
30 & S. viridis & V & 2.63 & 5.3 & 12 & $<8$ & $<8$ & 9 \\
\hline
\end{tabular}

${ }^{a}$ IZD: Inhibition zone diameter (the concentration of the extract at $50 \mathrm{mg} / \mathrm{mL}$ ); ${ }^{\text {b }}$ Part: The part of plant used for extraction (L: leaves; TBL: tender branches and leaves; WP: whole plant; V: vane); ${ }^{c}$ Weight: The weight of extract; ${ }^{\mathrm{d}}$ Ratio: The ratio of extract; ${ }^{\mathrm{e}} \mathrm{SA}$ : Staphylococcus aureus (ATCC 25923, MSSA); ${ }^{\mathrm{f}}$ EC: Escherichia coli (ATCC 25922); ${ }^{\mathrm{g}} \mathrm{PA}:$ Pseudomonas aeruginosa (ATCC 27853); ${ }^{\mathrm{h}}$ CA: Candida albicans (ATCC Y0109).

Table 4. Comparison of IZDs of the 21 extracts against MRSA and MSSA strains (IZD: mm) ${ }^{\mathrm{a}}$.

\begin{tabular}{lllll}
\hline No. & Extracts & MSSA & MRSA $_{\text {ave }} \pm$ SEM (n) $)^{\mathrm{b}}$ & $\triangle\left(\right.$ MSSA-MRSA $\left._{\text {ave }}\right)$ \\
\hline 1 & M. yunnanensis & 24 & $17.5 \pm 1.04(4)$ & 6.5 \\
2 & S. arborescens & 24 & $18.8 \pm 2.06(4)$ & 5.2 \\
3 & C. austroglauca & 23 & $18.5 \pm 1.85(4)$ & 4.5 \\
4 & M. hongheensis & 21 & $15.8 \pm 1.65(4)$ & 5.2 \\
5 & B. hancei & 19 & $16.0 \pm 1.63(4)$ & 3.0 \\
6 & S. sinensis & 18 & $14.3 \pm 1.31(4)$ & 3.7 \\
7 & E. daneillii & 18 & $13.8 \pm 1.31(4)$ & 4.2 \\
8 & M. squamulata & 17 & $14.5 \pm 0.87(4)$ & 2.5 \\
9 & G. morella & 17 & $15.7 \pm 0.67(3)$ & 1.3 \\
10 & C. orchioides & 16 & $17.3 \pm 0.95(4)$ & -1.3 \\
11 & E. fortunei & 15 & $15.5 \pm 1.55(4)$ & -0.5 \\
12 & A. nepalensis & 14 & $17.0 \pm 1.41(4)$ & -3.0 \\
13 & I. simonsii & 13 & $12.8 \pm 1.11(4)$ & 0.2 \\
14 & B. balsamifer & 12 & $7.0 \pm 1.22(4)$ & 5.0 \\
15 & S. viridis & 12 & $11.0 \pm 1.68(4)$ & 1.0 \\
16 & S. tamariscina & 12 & $11.0 \pm 1.00(4)$ & 1.0 \\
17 & M. salicina & 12 & $9.7 \pm 0.33(3)$ & 2.3 \\
18 & P. molle & 11 & $15.8 \pm 1.38(4)$ & -4.8 \\
19 & C. orbiculatus & 11 & $9.5 \pm 0.50(4)$ & 1.5 \\
20 & E. Burm & 10 & $13.0 \pm 2.08(3)$ & -3.0 \\
21 & C. prainii & 10 & $9.0 \pm 1.00(2)$ & 1.0 \\
\hline
\end{tabular}

${ }^{\mathrm{a}}$ IZD: Inhibition zone diameter (the concentration of the extract at $50 \mathrm{mg} / \mathrm{mL}$ ); ${ }^{\mathrm{b}}$ Number of MRSA isolates.

Table 5. Anti-MRSA activity of the extracts to the zone of inhibition against MSSA $\geq 10 \mathrm{~mm}$ (MIC/MBC: $\mu \mathrm{g} / \mathrm{mL}$ ).

\begin{tabular}{lllllllll}
\hline \multirow{2}{*}{ No. } & $\begin{array}{l}\text { Extracts and } \\
\text { Vancomycin }\end{array}$ & \multirow{2}{*}{ Activity } & \multirow{2}{*}{ MSSA } & $\begin{array}{l}\text { MRSA } \\
\mathbf{0 8 2}\end{array}$ & $\begin{array}{l}\text { MRSA } \\
\mathbf{0 9 2}\end{array}$ & $\begin{array}{l}\text { MRSA } \\
\mathbf{1 8 9}\end{array}$ & $\begin{array}{l}\text { MRSA } \\
\mathbf{1 4 4}\end{array}$ & $\begin{array}{l}\text { MRSA } \\
\mathbf{3 2 1}\end{array}$ \\
\hline \multirow{2}{*}{1} & \multirow{2}{*}{ M. yunnanensis } & MIC & 32 & 8 & 32 & 64 & 16 & 32 \\
& & MBC & 128 & 64 & 256 & 128 & 64 & 256 \\
\multirow{2}{*}{2} & \multirow{2}{*}{ S. arborescens } & MIC & 64 & 64 & 64 & 64 & 16 & 32 \\
& & MBC & 256 & 256 & 256 & 256 & 128 & 128 \\
\multirow{2}{*}{3} & \multirow{2}{*}{ C. austroglauca } & MIC & 64 & 64 & 64 & 64 & 32 & 16 \\
& & MBC & 256 & 128 & 512 & 256 & 128 & 256 \\
\hline
\end{tabular}


Table 5. Cont.

\begin{tabular}{|c|c|c|c|c|c|c|c|c|}
\hline No. & $\begin{array}{l}\text { Extracts and } \\
\text { Vancomycin }\end{array}$ & Activity & MSSA & $\begin{array}{l}\text { MRSA } \\
082\end{array}$ & $\begin{array}{l}\text { MRSA } \\
092\end{array}$ & $\begin{array}{l}\text { MRSA } \\
189 \\
\end{array}$ & $\begin{array}{l}\text { MRSA } \\
144 \\
\end{array}$ & $\begin{array}{l}\text { MRSA } \\
321 \\
\end{array}$ \\
\hline \multirow{2}{*}{4} & \multirow{2}{*}{ M. hongheensis } & MIC & 32 & 128 & 8 & 32 & 16 & 16 \\
\hline & & $\mathrm{MBC}$ & 128 & 512 & 32 & 128 & 64 & 64 \\
\hline \multirow{2}{*}{5} & \multirow{2}{*}{ B. hancei } & MIC & 64 & 64 & 32 & 64 & 32 & 64 \\
\hline & & $\mathrm{MBC}$ & 128 & 256 & 256 & 128 & 128 & 256 \\
\hline \multirow{2}{*}{6} & \multirow{2}{*}{ E. daneillii } & MIC & 32 & 32 & 32 & 32 & 32 & 64 \\
\hline & & $\mathrm{MBC}$ & 64 & 256 & 128 & 256 & 256 & 256 \\
\hline \multirow{2}{*}{7} & \multirow{2}{*}{ S. sinensis } & MIC & 32 & 64 & 32 & 32 & 32 & 16 \\
\hline & & $\mathrm{MBC}$ & 128 & 256 & 256 & 64 & 128 & 64 \\
\hline \multirow{2}{*}{8} & \multirow{2}{*}{ G. morella } & MIC & 32 & 32 & 64 & 32 & 16 & 32 \\
\hline & & $\mathrm{MBC}$ & 256 & 256 & 256 & 128 & 64 & 128 \\
\hline \multirow{2}{*}{9} & \multirow{2}{*}{ M. squamulata } & MIC & 64 & 32 & 64 & 32 & 64 & 32 \\
\hline & & $\mathrm{MBC}$ & 256 & 128 & 256 & 128 & 256 & 128 \\
\hline \multirow{2}{*}{10} & \multirow{2}{*}{ C. orchioides } & MIC & 512 & 256 & 512 & 512 & 512 & 256 \\
\hline & & $\mathrm{MBC}$ & 1,024 & 1,024 & $>2,048$ & $>2,048$ & 512 & 1024 \\
\hline \multirow{2}{*}{11} & \multirow{2}{*}{ E.fortunei } & MIC & 512 & 512 & 512 & 512 & 512 & 512 \\
\hline & & $\mathrm{MBC}$ & $>2,048$ & $>2,048$ & 1,024 & $>2,048$ & $>2,048$ & $>2,048$ \\
\hline \multirow{2}{*}{12} & \multirow{2}{*}{ A. nepalensis } & MIC & 1024 & 512 & 256 & 512 & 512 & 256 \\
\hline & & $\mathrm{MBC}$ & $>2,048$ & 1,024 & 1,024 & $>2,048$ & $>2,048$ & 1,024 \\
\hline \multirow{2}{*}{13} & \multirow{2}{*}{ I. simonsii } & MIC & 512 & 512 & 512 & 1,024 & 1,024 & 1,024 \\
\hline & & $\mathrm{MBC}$ & 1,024 & $>2,048$ & $>2,048$ & $>2,048$ & $>2,048$ & $>2,048$ \\
\hline \multirow{2}{*}{14} & \multirow{2}{*}{ B. balsamifer } & MIC & 256 & 256 & 256 & 64 & 256 & 128 \\
\hline & & $\mathrm{MBC}$ & 1,024 & 256 & 1,024 & 256 & 1,024 & 256 \\
\hline \multirow{2}{*}{15} & \multirow{2}{*}{ M. salicina } & MIC & 512 & 512 & 1,024 & 512 & 512 & 512 \\
\hline & & $\mathrm{MBC}$ & 1,024 & $>2,048$ & $>2,048$ & $>2,048$ & 1,024 & $>2,048$ \\
\hline \multirow{2}{*}{16} & S wirridis & MIC & 256 & 128 & 64 & 128 & 128 & 128 \\
\hline & S. viridis & $\mathrm{MBC}$ & 1,024 & 512 & 256 & 512 & 512 & 512 \\
\hline 17 & S tamariscing & MIC & 512 & 1,024 & 1,024 & 1,024 & 1,024 & 1,024 \\
\hline 17 & S. tamarlscina & $\mathrm{MBC}$ & 1,024 & $>2,048$ & $>2,048$ & 2,048 & $>2,048$ & $>2,048$ \\
\hline 18 & & MIC & 1,024 & 1,024 & 512 & 512 & 1,024 & 512 \\
\hline 18 & C. orbiculatus & $\mathrm{MBC}$ & $>2,048$ & $>2,048$ & 1,024 & 1,024 & $>2,048$ & 1,024 \\
\hline 10 & $P$ mollo & MIC & 512 & 256 & 256 & 256 & 256 & 256 \\
\hline 19 & P.molle & $\mathrm{MBC}$ & $>2,048$ & 1,024 & 1,024 & 1,024 & 1,024 & 1,024 \\
\hline 20 & & MIC & 1,024 & 1,024 & 1,024 & 1,024 & 1,024 & 2,048 \\
\hline 20 & C.prainil & $\mathrm{MBC}$ & $>2,048$ & $>2,048$ & 2,048 & $>2,048$ & $>2,048$ & $>2,048$ \\
\hline & & MIC & 512 & 512 & 1,024 & 1,024 & 1,024 & 1,024 \\
\hline 21 & E.Burm & $\mathrm{MBC}$ & $>2,048$ & 1,024 & $>2,048$ & $>2,048$ & $>2,048$ & $>2,048$ \\
\hline 1 & Vancomucin & MIC & 1 & 1 & 1 & 1 & 1 & 1 \\
\hline 1 & vancomycin & $\mathrm{MBC}$ & 2 & 2 & 2 & 2 & 2 & 2 \\
\hline
\end{tabular}

\subsection{Discussion}

A collection of 30 Chinese medicinal plants were evaluated for their in vitro antimicrobial effects, especially their anti-MRSA potentials. Most of the plants with anti-infective effects related to skin 
infections have been indicated in various works of Chinese herbal medicines either by themselves or the different species belonging to the same botanical genus, such as the indication of carbuncle which means a severe abscess or multiple boil in the skin, typically infected with S. bacteria (Table 1) [6-11]. The antimicrobial screening of all the plant species is being reported for the first time to the best of our knowledge, especially the anti-MRSA activities.

The $80 \%$ ethanol extracts of the 30 Chinese medicinal plant extracts were initially subjected to the screening of their antimicrobial activities against MSSA and other conventional standard strains of Escherichia coli (ATCC 25922), Pseudomonas aeruginosa (ATCC 27853) and Candida albicans (ATCC Y0109). Their activities as IZDs $(\mathrm{mm})$ at a concentration of $50 \mathrm{mg} / \mathrm{mL}$ ranged from $<8$ to 24, of which 21 extracts showed IZDs $\geq 10 \mathrm{~mm}$ against MSSA (Table 1). The results were in agreement with the literature record of traditional usages (Table 3) [6-11]. Judging from the IZD values, the extracts were generally more active against the Gram positive pathogen (MSSA) than against Gram negative (E. coli and P. aeruginosa) and fungal pathogens (C. albicans). The additional permeability barrier caused by the outer lipopolysaccharide layer in Gram negative bacteria makes them more resistant to plant natural products, as has been demonstrated previously [13,14]. Therefore, finding out anti-staphylococcal natural products from plant materials is much easier and is proved in the previous reports [15-18].

The tested MRSA strains were seven multi-drug resistant clinical isolates (Table 2). They were used for the further assay of their susceptibilities to the 21 extracts (Table 4). It is interesting that these extracts which were active against MSSA also showed more or less activity against MRSA with IZD values ( $\mathrm{mm}$ ) ranging between 9.0 and 18.8 and this has been noted previously [15-19]. The differences were from -3.0 to $6.5 \mathrm{~mm}$ in this study (Table 4).

Anti-MRSA potency of MICs/MBCs $(\mu \mathrm{g} / \mathrm{mL})$ of the 21 extracts was determined as 8-2,048/32->2,048 (Table 5). Comparison of the values of IZD and MIC for a same extract in Tables 4 and 5, they were not always positively correlated. As different sample concentrations will produce different IZDs, the real inhibitory potency of a sample should be judged by its MIC. This should be noted for future studies.

From the phytochemical constitution of the 30 plant materials listed in Table 1, it is found that most of the active extracts contained tannins, (poly)phenols (including flavonoids, lignans and coumarins), terpenoids or alkaloids which have been reported [18,20-25].

The phytochemicals, including polyphenols and antimicrobial activity of Mallotus species have been reviewed [26,27]. Rottlerin from M. philippinensis exhibited potent bactericidal activity with an MBC value of 3.12-6.25 $\mu \mathrm{g} / \mathrm{mL}$ against several clinical H. pylori isolates [28]. Garcinia genus is rich in caged xanthones [29]. Morellin isolated from gamboge ( $G$. hanburyi, tenghuang in Chinese) was reported as an antibiotic principle in 1954 [30]. The anti-MRSA activity of morellin and other caged xanthones from Gamboge, G. morella and other Garcinia species have also reported [31,32]. Two antifungal compounds ulopterol (a coumarin) and a quinolone alkaloid 4-Methoxy-1-methyl-3(2'-S-hydroxy-3'-ene-butyl)-2-quinolone were isolated from Skimmia laureola, a Pakistani medicinal plant [33], which were similar to our results of the inhibition of $S$. arborescens against $C$. albicans (Table 3). However, no active antimicrobial compounds from the other plant materials have been isolated up to now, especially the anti-MRSA compounds from the most active extracts of $S$. sinensis, E. daneillii, M. squamulata, and B. hancei, with MICs at 8-64 $\mu \mathrm{g} / \mathrm{mL}$. We are continuing our search for the corresponding phytochemicals from these plants and their further systematic anti-MRSA properties. 


\section{Experimental}

\subsection{Plant Materials}

The selected 30 Chinese medicinal plant samples were collected from the tropical mountain forests of southeastern Yunnan Province of China, at altitudes of 1,500-3,074 m in June 2010. They were identified by Y.M. Shui at the Kunming Institute of Botany (KIB); the Chinese Academy of Sciences. Voucher specimens are preserved at the herbarium of KIB [34]. The names of species/family (specimen No.) were as the following: Alnus nepalensis D. Don./Betulaceae (KUN 35); Blumea balsamifer (Linn.) D.C./Asteraceae (KUN 21); Brandisia hancei Hook. f./Scrophulariaceae (YFS 6); Carex prainii C.B. Clarke/Cyperaceae (KUN 11133); Cayratiy japonica (Thunb) Gngacp.var.japonica/Vitaceae (YFS 531); Celastrus orbiculatus Thunb./Celastraceae (KUN 110); Curculigo orchioides Gaertn./Hypoxidaceae (KUN 1151); Cyclobalanopsis austroglauca Y.T. Chang/Fagaceae (SWFC 851222); Embelia Burm f./Myrsinaceae (KUN355); Euonymus fortunei (Turcz.); Hand. Mazz./Celastraceae (YUKU (s.n)); Euonymus laxiflorus Charmp.ex.Benth./Celastraceae (YCP 851027); Evodia daneillii (Benn) Hemsl./Rutaceae (YFS 663); Garcinia morella Desr./Clusiaceae (YCP 851757); Illicium simonsii Maxim./I11 iciaceae (IBSC 125); Kadsura angustifolia A.C. Smith/Schisandraceae (KUN 4480); Litsea lancifolia (Roxb. ex. Nees); Benth. et. Hook. f. ex. F./Lauraceae (KUN 23); Machilus salicina Hance./Lauraceae (YUKU 1535); Mallotus yunnanensis Pax et. Hoffm./Euphorbiaceae (YFS 1144); Manglietia hongheensis Y.m Shui et. W.H. Chen./Magnoliaceae (KUN 262); Meliosma squamulata Hance./Lauraceae (KUN 2411); Oenanthe javanica (B1.)DC./Umbellifera (KUN 214); Polygonum molle D. Don./Polygonaceae (KUN 367); Pyrularia edulis (Wall.) A.D.C./Santalaceae (KUN 60002100); Ranunculus japonicus Thumb./Ranunculaceae (KUN 2398); Schima sinensis (Hemsl. et. Wils) Airy-shaw./Theaceae (KUN158); Schisandra viridis A.c.Smith./Schisandraceae (YFS); Scurrula parasitica Linn.var. parasitica./Loranthaceae (YFS 327); Selaginella tamariscina (Seauv.) Spring./Selaginellaceae (YCP 85937); Skimmia arborescens Anders./Rutaceae (PE 100260); Sophora davidii (Franch.) Skeels./Leguminosae (YCP 851049). The traditional indications and phytochemical constitutions were listed in Table 1.

\subsection{Microbial Strains and Culture Media}

Standard bacterial and fungal strains, i.e., Staphylococcus aureus (ATCC 25923, MSSA), Escherichia coli (ATCC 25922), Pseudomonas aeruginosa (ATCC 27853) and Candida albicans (ATCC Y0109) were provided by the National Institute for the Control of Pharmaceutical and Biological Products (NICPBP, China). Clinical MDR MRSA strains of MRSA 8, MRSA 82, MRSA 92, MRSA 123, MRSA 144, MRSA 189 and MRSA 321 were clinical isolates from infectious samples of critically ill patients in Kunming General Hospital $(\mathrm{KGH})$. Pathogen purification and identification (including colonial morphology, Gram staining and coagulase testing) were conducted in our clinical microbiology laboratory and further confirmed by standard cefoxitin disk diffusion test following the Clinical and Laboratory Standards Institute (CLSI) guidelines [15,35,36]. ATCC 25923 was used as the control strain. Vancomycin (Eli Lilly Japan K.K., Seishin Laboratories) was used as a control anti-MRSA agent. Standard Mueller-Hinton agar and broth (MHA and MHB) and Sabouraud agar 
(Tianhe Microbial Agents Co., Hangzhou, China) were used as the bacterial and fungal culture media, respectively. The antibacterial agents-susceptibility testing results of MRSA strains were shown in Table 2.

\subsection{Extract Preparation}

The 30 samples of the air-dried and ground plant materials $(50 \mathrm{~g})$ were macerated with $80 \%$ ethanol $(500 \mathrm{~mL})$ for 7 days, filtered and the mare was further macerated twice with the same solvent overnight and filtered after being sonicated for $30 \mathrm{~min}$. The filtrates were combined and the solvent was evaporated at $40^{\circ} \mathrm{C}$ in vacuum to afford each of the plant extract (Table 3 ).

\subsection{Antimicrobial Screening}

The ethanol extracts of the 30 plants were primarily subjected to susceptibility screening against standard microbial strains according to the agar diffusion method on MHA (for the bacteria) or SA (for C. albicans) plates. The sample extracts $(50 \mathrm{mg} / \mathrm{mL}$ in dimethyl sulfoxide) were pipetted into $6 \mathrm{~mm}$ (in diameter) holes punched in the agar of prepared agar plates, plating with inoculums of $1.5 \times 10^{8} \mathrm{CFU} / \mathrm{mL}$ for bacteria and $5 \times 10^{5} \mathrm{CFU} / \mathrm{mL}$ for $C$. albicans in advance, respectively and incubated at $35{ }^{\circ} \mathrm{C}$ (for C. albicans at $28^{\circ} \mathrm{C}$ ) for $24 \mathrm{~h}$, measured and recorded the IZDs [15-17,37]. The solvent value was deducted accordingly to get final results of activity. All experiments were carried out in triplicate. The test results were interpreted based on IZD as $<10 \mathrm{~mm}$ for the resistance, $=10 \mathrm{~mm}$ for the mildly susceptible, $11-15 \mathrm{~mm}$ for moderately susceptible, $\geq 16 \mathrm{~mm}$ for the highly susceptible (Table 2 ). The 21 extracts with IZDs $\geq 10 \mathrm{~mm}$ against MSSA were further subjected to the assay of their IZDs against MRSA strains (Table 3).

\subsection{MICs and MBCs Assaying}

The extracts with IZDs $\geq 10 \mathrm{~mm}$ against MSSA were further subjected to the assay of their IZDs and minimal inhibitory concentrations and minimal bactericidal concentrations (MICs/MBCs) against MRSA strains by serial dilution method according to the procedures reported previously [15-17,38,39]. Briefly, MICs/MBCs were determined by the standardized broth (using MHB) microdilution techniques with starting inoculums of $5 \times 10^{5} \mathrm{CFU} / \mathrm{mL}$ for the bacteria according to CLSI (formally NCCLS) guidelines and incubated at $35{ }^{\circ} \mathrm{C}$ for $24 \mathrm{~h}$. For the MBCs assaying, $0.1 \mathrm{~mL}$ aliquots from drug dilution wells with visual growth inhibition were plated onto MHA media. The lowest drug concentration that yielded three or fewer microorganism colonies was recorded as the MBC. They were determined in triplicate, with concentrations ranging up to $2048 \mu \mathrm{g} / \mathrm{mL}$ for all the extracts (Table 5).

\section{Conclusions}

The screening of in vitro antimicrobial activity of the ethanol extracts from 30 Chinese medicinal plants led to the confirmation of 21 extracts displaying both anti-MSSA and MRSA effects with various levels of potency which were in good agreement with their TCM indications of skin infections and modern phytochemical constituents, with M. yunnanensis and S. arborescens extracts being the most active against MRSA. The combination of TCM indications and phytochemical profiles is a promising approach to the search of anti-MRSA plant natural products. 


\section{Acknowledgements}

This work was supported by the National Natural Science Foundation of China (NSFC 81073126, 81173504) and the supporting funds of the Department of Health, Chengdu Military Region, PLA of China (MB09030) and Yunnan Province of China (2008PY001).

\section{References and Notes}

1. Pantosti, A.; Venditti, M. What is MRSA? Eur. Respir. J. 2009, 34, 1190-1196.

2. Daniel, S.F.; Norman, R. The value of plants used in traditional medicine for drug discovery. Environ. Health Perspect. 2001, 109, 69-75.

3. Mahady, G.B. Medicinal plants for the prevention and treatment of bacterial infections. Curr. Pharm. Des. 2005, 11, 2405-2427.

4. Gibbons, S. Phytochemicals for bacterial resistance-Strengths, weaknesses and opportunities. Planta Med. 2008, 74, 594-602.

5. Sibanda, T.; Okoh, A.I. The challenges of overcoming antibiotic resistance: Plant extracts as potential sources of antimicrobial and resistance modifying agents. Afr. J. Biotechnol. 2007, 6, 2886-2896.

6. The Compiler Group. Shen Nong's Materia Medica; Tianjin Ancient Books Publishing House: Tianjin, China, 2009.

7. Li, S.Z. Compendium of Materia Medica; Heilongjiang Fine Arts Publishing House: Harbin, China, 2009.

8. Jiangsu New Medical College. Dictionary of Chinese Materia Medica; Shanghai Science and Technology Press: Shanghai, China, 1977.

9. State Administration of Traditional Chinese Medicine. Chinese Materia Medica; Shanghai Science and Technology Press: Shanghai, China, 1999.

10. The Compiler Group. Compilation of Chinese herbal medicine; People's Medical Publishing House: Beijing, China, 1975.

11. The Compiler Group. Selected Yunnan Traditional Chinese Herbs; Tianjin People's Press: Tianjin, China, 1970.

12. Name of disease "Chuang-Yang". In Chinese-English Pocket Dictionary of Traditional Chinese Medicine; Zhang, T.M., Jia, B., Liao, C.D., Eds.; People's Medical Publishing House: Beijing, China, 2003; pp. 47-48.

13. Tegos, G.; Stermitz, F.R.; Lomovskaya, O.; Lewis, K. Multidrug pump inhibitors uncover remarkable activity of plant antimicrobials. Antimicrob. Agents Chemother. 2002, 46, 3133-3141.

14. Kumar, V.P.; Chauhan, N.S.; Padh, H.; Rajani, M. Search for antibacterial and antifungal agents from selected Indian medicinal plants. J. Ethnopharmacol. 2006, 107, 182-188.

15. Zuo, G.Y.; Wang, G.C.; Zhao, Y.B.; Xu, G.L.; Hao, X.Y.; Han, J.; Zhao, Q. Screening of Chinese medicinal plants for inhibition against clinical isolates of methicillin-resistant Staphylococcus aureus (MRSA). J. Ethnopharmacol. 2008, 120, 287-290.

16. Zuo, G.Y.; Wang, G.C.; Xu, G.L.; Yu, W.; Peng, H.; Kuang, W.X. Screening of anti-MRSA activity from 30 Chinese herbage medicine extracts. Chin. J. Mod. Appl. Pharm. 2006, 23, 293-295. 
17. Zuo, G.Y.; Yu, W.; Xu, G.L.; Wang, G.C.; Peng, H.; Kuang, W.X. Screen of anti-MRSA activity from 18 Chinese herbage medicine extracts. China Pharm. 2005, 8, 606-608.

18. Gibbons, S. Anti-staphylococcal plant natural products. Nat. Prod. Rep. 2004, 21, 263-277.

19. Weckesser, S.; Engel, K.; Simon-Haarhaus, B.; Wittmer, A.; Pelz, K.; Schempp, C.M. Screening of plant extracts for antimicrobial activity against bacteria and yeasts with dermatological relevance. Phytomedicine 2007, 14, 508-516.

20. Cowan, M.M. Plant products as antimicrobial agents. Clin. Microbiol. Rev. 1999, 12, 564-582.

21. Cushnie, T.P.; Lamb, A.J. Antimicrobial activity of flavonoids. Int. J. Antimicrob. Agents 2005, $26,343-356$.

22. An, J.; Zuo, G.Y.; Hao, X.Y.; Wang, G.C.; Li, Z.S. Antibacterial and synergy of a flavanonol rhamnoside with antibiotics against clinical isolates of methicillin-resistant Staphylococcus aureus (MRSA). Phytomedicine 2011, 18, 990-993.

23. Zuo, G.Y.; Meng, F.Y.; Hao, X.Y.; Zhang, Y.L.; Wang, G.C.; Xu, G.L. Antibacterial alkaloids from Chelidonium majus Linn (Papaveraceae) against clinical isolates of methicillin-resistant Staphylococcus aureus. J. Pharm. Pharm. Sci. 2008, 11, 90-94.

24. Zuo, G.Y.; Li, Y.; Wang, T.; Han, J.; Wang, G.C.; Zhang, Y.L.; Pan, W.D. Synergistic antibacterial and antibiotic effects of bisbenzylisoquinoline alkaloids on clinical isolates of methicillin-resistant Staphylococcus aureus (MRSA). Molecules 2011, 16, 9819-9826.

25. Meng, F.Y.; Zuo, G.Y.; Hao, X.Y.; Wang, G.C.; Xiao, H.T.; Zhang, J.Q.; Xu, G.L. Antifungal activity of the benzocphenanthridine alkaloids from Chelidonium majus Linn against resistant clinical yeast isolates. J. Ethnopharmacol. 2009, 125, 494-496.

26. Rivière, C.; Nguyen, T.H.V.; Tran, H.Q.; Chataigné, G.; Nguyen, H.N.; Dejaegher, B.; Tistaert, C.; Nguyen, T.K.T.; Vander, H.Y.; Chau, V.M.; et al. Mallotus species from Vietnamese mountainous areas: Phytochemistry and pharmacological activities. Phytochem. Rev. 2010, 9, 217-253.

27. Riviere, C.; Hong, V.N.; Pieters, L.; Dejaegher, B.; Heyden, Y.V.; Van, M.C.; Quetin-Leclercq, J. Polyphenols isolated from antiradical extracts of Mallotus metcalfianus. Phytochemistry 2009, 70, 86-94.

28. Zaidi, S.F.; Yoshida, I.; Butt, F.; Yusuf, M.A.; Usmanghani, K.; Kadowaki, M.; Sugiyama, T. Potent bactericidal constituents from Mallotus philippinensis against clarithromycin and metronidazole resistant strains of Japanese and Pakistani Helicobacter pylori. Biol. Pharm. Bull. 2009, 32, 631-636.

29. Han, Q.B.; Xu, H.X. Caged Garcinia xanthones: Development since 1937. Curr. Med. Chem. 2009, 16, 3775-3796.

30. Narasimha Rao, P.L.; Krishna Murthy, D.V.; Verma, S.C.L. Antibiotic principles of garcinia morella. Constitution of morellin. Naturwissenschaften 1954, 41, 66-67.

31. Sukpondma, Y.; Rukachaisirikul, V.; Phongpaichit, S. Antibacterial caged-tetraprenylated xanthones from the fruits of Garcinia hanburyi. Chem. Pharm. Bull. 2005, 53, 850-852.

32. Chantarasriwong, O.; Batova, A.; Chavasiri, W.; Theodorakis, E.A. Chemistry and biology of the caged Garcinia xanthones. Chemistry 2010, 16, 9944-9962. 
33. Ahmad, K.F.; Sultana, N. Studies on bioassay directed antifungal activity of medicinal plants Calotropis procera, Skimmia laureola, Pettophorum pterocarpum and two pure natural compounds ulopterol and 4-methoxy-1-methyl-3-(2'-S-hydroxy-3'-ene-butyl)-2-quinolone. J. Chem. Soc. Pakistan 2003, 25, 328-330.

34. Seed Plants of Honghe Region in SE Yunnan, China; Shui, Y.M., Ed.; Yunnan Science and Technology Press: Kunming, China, 2003.

35. Clinical and Laboratory Standards Institute. Performance Standards for Antimicrobial Disk Susceptibility Tests, Approved Standard, Document M2-A9, 9th ed.; CLSI: Wayne, PA, USA, 2006.

36. Clinical and Laboratory Standards Institute. Performance Standards for Antimicrobial Susceptibility Testing-17th Informational Supplement, Approved Standard, M100-S17; CLSI: Wayne, PA, USA, 2007.

37. Khan, R.; Islam, B.; Akram, M.; Shakil, S.; Ahmad, A.; Ali, S.M.; Siddiqui, M.; Khan, A.U. Antimicrobial activity of five herbal extracts against multi drug resistant (MDR) strains of bacteria and fungus of clinical origin. Molecules 2009, 14, 586-597.

38. Clinical and Laboratory Standards Institute. Methods for Dilution Antimicrobial Susceptibility Tests for Bacteria that Grow Aerobically, Approved Standard, Document M7; CLSI: Wayne, PA, USA, 2006.

39. National Committee for Clinical Laboratory Standards. Methods for Determining Bactericidal Activity Antimicrobial Agents, Approved Guidelines, Document M26-A; CLSI (formerly NCCLS): Wayne, PA, USA, 1999.

Sample Availability: Not available.

(C) 2012 by the authors; licensee MDPI, Basel, Switzerland. This article is an open access article distributed under the terms and conditions of the Creative Commons Attribution license (http://creativecommons.org/licenses/by/3.0/). 